\title{
Zukünftige Grundwasserforschung - Was sind unsere Aufgaben?
}

\author{
Mario Schirmer \\ Online veröffentlicht: 12.8.2008 \\ (C) Springer-Verlag 2008
}

Liebe Leserinnen und Leser!

In der FH-DGG und mit der Zeitschrift

Grundwasser haben wir es uns zu einer dervordringlichstenAufgaben gemacht, die Brücke zwischen Wissenschaft, Behörden und Praxis zu schlagen. Da ich mich gerade in ein neues Wissenschaftsumfeld begeben habe und das Privileg genieße, alte Forschungsideen zu überdenken und neue zu definieren, dachte ich, es wäre gut, einige meiner Gedanken mit Ihnen/mit Euch zu teilen. Ausschlaggebend für das Schreiben dieses Editorial war jedoch, dass ich den Bericht des „Intergovernmental Panel on Climate Change" (IPCC): "Climate Change 2007" eingehend studiert habe. Für all jene, die diesen noch nicht gelesen haben, empfehle ich den „Climate Change 2007: Synthesis Report" (zu finden z. B. unter http://www. ipcc.ch/). Für einen Geowissenschaftler und eigentlich jeden Mitbürger ist dieser Bericht hoch interessant und erschütternd zugleich. Zwar bestehen noch viele Unsicherheiten und Datenlücken, aber die „Beweislage“, dass wir gewaltigen globalen Veränderungen entgegen gehen, ist schon erdrückend. Diese werden auf jeden Fall den Wasserzyklus nachhaltig beeinflussen und große ökonomische sowie wissenschaftlich-technische Anstrengungen auf vielen Gebieten fordern.

Es ist unsere Aufgabe, dass wir uns mit der Qualität und der Quantität der Was-

\footnotetext{
Mario Schirmer $(\square)$

Abteilung Wasserressourcen und Trinkwasser, Urbane und Alpine Hydrogeologie, EAWAG,

Überlandstr. 133, 8600 Dübendorf, Schweiz

E-Mail: mario.schirmer@eawag.ch
}

serressourcen beschäftigen. Die Versorgung der Menschen mit Wasser zu bezahlbaren Preisen ist gewissermaßen unsere Berufung. Zumindest in meinen Augen ist das unsere Verantwortung der Gesellschaft gegenüber.

Viele Wissenschaftlerinnen und Wissenschaftler haben sich über die letzten drei Jahrzehnte intensiv mit industriell kontaminierten Grundwasserleitern beschäftigt. Die Schadstoffpalette ist breit. Sie reicht von chlorierten und polyzyklischen aromatischen Kohlenwasserstoffen (CKW, PAK) über Benzin, Mineralölkohlenwasserstoffen (MKW) und Methyl-tertiär-butyl-ether (MTBE), um nur einige zu nennen. Auch landwirtschaftliche Schadstoffe, wie Pestizide, Nitrat und Phosphat haben viel Beachtung gefunden. Selbstverständlich gibt es noch die eine oder andere spannende Frage zu klären oder den einen oder anderen besonders komplizierten Standort zu bearbeiten. Aber es gibt meiner Meinung nach eine Vielzahl von exzellent arbeitenden Ingenieurbüros, die viele Erkenntnisse und Erfahrungen aus der Wissenschaft in die Praxis getragen haben. Außerdem verzeichnen wir bereits viele ermutigende Ansätze, wie Behörden neue wissenschaftliche Konzepte in ihre Verordnungen einfließen lassen und kreativ im Rahmen der Gesetze interpretieren.

Die Wissenschaft ist gefordert, die knappen Forschungs-Euros, bzw. Franken für die Schweizer Kollegen, intelligent einzusetzen. So ist es an der Zeit, sich mit den Schadstoffen der Zukunft zu beschäftigen. Es ist schließlich besser, vorsorgend zu handeln, als ständig Nachsorge zu betreiben. Dabei kommt natürlich sofort die Frage auf: Was sind denn die Schadstoffe der Zukunft? Das ist nicht einfach zu beantworten, da am Beginn der Forschung die Frage der Relevanz aufgrund des geringen Kenntnisstandes und der komplexen Interaktionen meist nicht geklärt werden kann. Wie viele wissen, bin ich der Meinung, dass Pharmaka, Substanzen in Körperpflegemitteln (pharmaceuticals and personal care products - PPCP) und endokrin wirksame Substanzen den Ökosystemen und uns Menschen potenziell selbst bei geringen Konzentrationen Schaden zufügen können. Eine wachsende Zahl von biologischen Studien belegt dies. Deshalb haben wir uns über die letzten Jahre auch besonders mit der urbanen Hydrogeologie beschäftigt, wo viele dieser Stoffe in großer Anzahl und in komplexen Gemischen anzutreffen sind (siehe auch Grundwasser-Heft 3/2007).

Aber selbstverständlich gibt es auch andere Stoffe, die in Zukunft Forschungsgegenstand werden könnten. Wir müssen als Wissenschaftler vorausschauen und die potenziellen Probleme erkennen, bevor die Sanierung der Schäden Millionen oder Milliarden verschlingt. Diese Mittel sind auf jeden Fall besser im Bildungssystem aufgehoben oder können sinnvoller für andere gesellschaftliche Aufgaben eingesetzt werden.

Über die letzten Jahre ist jedoch auch klar geworden, dass wir uns als Wasserforscher insbesondere auf dem Gebiet der Auswirkungen des globalen Wandels für die Quantität und Qualität der Wasserressourcen engagieren sollten. Dabei geht es oft um grundlegende 
Fragestellungen, wie z.B. der Wasserkreislauf den globalen Wandel beeinflusst. Eigentlich sehr verwunderlich ist, dass in den vielen Klimamodellen, die für den IPCC 2007-Bericht verwendet wurden, für die untere Randbedingung, die Erdoberfläche, nur sehr vereinfachte Annahmen getroffen werden, z.B. in Bezug auf Energiebilanz und Verdunstung. Das Grundwasser ist dort nicht berücksichtigt. Meiner Meinung nach müssen wir das ändern und die dafür notwendigen Arbeiten leisten. Aber natürlich müssen da Kräfte gebündelt werden. Es müssen vor allem Felddaten in verschiedenen Höhenlagen und Temperaturregimen gewonnen werden und wir benötigen Langzeituntersuchungen. Auch bin ich der Meinung, dass wir die Prozesse der Grundwasserneubildung noch nicht vollständig verstanden haben und dort noch Forschungsbedarf besteht. Auf der anderen Seite ist es erfreulich, die wachsende Anzahl an Arbeiten an der Schnittstelle Grundwasser - Oberflächenwasser zu sehen. All dieses Verständnis ist notwendig, damit wir belastbare Szenarien für die Klimamodellierung zur Verfügung stellen können.

Für mich sehe ich aufgrund meines Standortwechsels jetzt die Aufgabe, mich verstärkt mit alpinen Lockergesteinsgrundwasserleitern $\mathrm{zu}$ beschäftigen. Diese Arbeiten decken natürlich nur einen Teil der insgesamt notwendigen Forschung ab. In diesem Zusammenhang möchte ich deshalb alle interessierten Personen aufrufen, verstärkt auf dem Gebiet des globalen Wandels zu forschen. Vielleicht hat die Randbedingung Grundwasser doch eine wesentlich größere Bedeutung, als wir heute glauben. Aber auf jeden Fall haben wir der Gesellschaft gegenüber die Verpflichtung, unsere begrenzten Forschungsmittel sehr klug einzusetzen und da ist es gut, einmal Zäsur zu machen und darüber nachzudenken, was die Gesellschaft in Zukunft von uns benötigt und was in den kommenden Jahren international wichtige Themen sein werden.

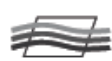

